Research Paper

\title{
Preoperative Albumin to Globulin Ratio (AGR) as Prognostic Factor in Renal Cell Carcinoma
}

\author{
Xiaobo He ${ }^{1,2^{*}}$, Shengjie Guo ${ }^{*}$, Dong Chen ${ }^{*}$, Guangwei Yang ${ }^{2}$, Xin Chen ${ }^{1}$, Yijun Zhang ${ }^{3}$, Qiuming He ${ }^{1}$, Zike

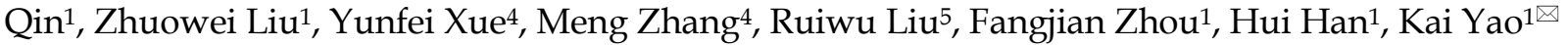 \\ 1. Department of Urology, Sun Yat-Sen University Cancer Center, State Key Laboratory of Oncology in South China, Collaborative Innovation Center for \\ Cancer Medicine, Guangzhou, China \\ 2. Department of Medical Oncology, the Fifth Affiliated Hospital of Sun Yat-Sen University, Zhuhai, China \\ 3. Department of Pathology, Sun Yat-sen University Cancer Center, Guangzhou, Guangdong, China \\ 4. Medicine school of Sun Yat-Sen University, Guangzhou, China \\ 5. Department of Biochemistry and Molecular Medicine, University of California Davis, Sacramento, California, United States of America \\ * These authors contributed equally to this work.
}

$\triangle$ Corresponding author: Kai Yao, Department of Urology, Sun Yat-sen University Cancer Center, 651 Dongfeng Road East, Guangzhou, Guangdong 510060, P.R. China. Email: yaokai@sysucc.org.cn.

(C) Ivyspring International Publisher. This is an open access article distributed under the terms of the Creative Commons Attribution (CC BY-NC) license (https://creativecommons.org/licenses/by-nc/4.0/). See http://ivyspring.com/terms for full terms and conditions.

Received: 2016.06.18; Accepted: 2016.10.15; Published: 2017.01.15

\begin{abstract}
Background: Malnutrition and systemic inflammatory response are frequently associated with prognosis in patients with several types of cancer, including renal cell carcinoma (RCC). The study is aimed to investigate the ability of preoperative serum albumin to globulin ratio (AGR) to predict the long-term mortality of RCC patients.

Methods: The study is a retrospective study of an unselected cohort of 895 RCC patients who underwent a curative radical or partial nephrectomy at the Department of Urology in the Sun Yat-Sen University Cancer Center between January 2000 and December 2012 and had documented preoperative serum total protein and albumin (ALB) levels. The preoperative AGR was calculated as the ratio of $A L B$ to (total protein-ALB) and its association with other clinical indices was assessed using survival analysis.

Results: Low preoperative AGR was associated with older population, lower hemoglobin, higher total protein, lower ALB, lower body mass index and advanced stage. The univariate and multivariate Cox analyses demonstrated that preoperative AGR was an independent prognostic indicator of overall survival (OS) (hazard ratio (HR): $0.63,95 \%$ confidence interval $(\mathrm{Cl}): 0.43$ to $0.93, \mathrm{P}=0.022$ ). In addition, patients with low preoperative AGR at pT1-2, pT3-4, pN0, pN1, pM0 and pM1 stages had significantly shorter OS than patients with high preoperative AGR.

Conclusion: Preoperative AGR is a proven objective, reproducible, inexpensive survival predictor of RCC patients following surgical resection and should be considered for routine clinical use.
\end{abstract}

Key words: Renal Cell Carcinoma; Albumin, Total Protein; Albumin to Globulin ratio.

\section{Introduction}

Renal cell carcinoma (RCC) is the most common malignant tumor of the kidney, with 66,800 and 62,700 estimated new cases occurring in China each year 1 and the United State in $2016^{2}$, respectively. Incidental and early stage tumors have been detected more frequently because of increased use of imaging techniques including ultrasound and computed tomography $(\mathrm{CT})$ in recent years ${ }^{3-4}$. Although more
RCC patients are diagnosed at early stage tumors, its mortality is still rising. Approximately $20 \%$ to $30 \%$ of patients with localized tumors after radical or partial nephrectomy will later develop metastatic disease ${ }^{5}$. As renal tumors are insensitive to radiotherapy and chemotherapy, majority of metastatic patients die. Although the TNM system proposed by UICC and AJCC ${ }^{6}$ and Fuhrman's Nuclear Grading system ${ }^{7}$ are 
commonly used for prognosis, they are not entirely reliable ${ }^{8}$. Other well-known prognostic factors are lymphocytic infiltration and histological subtype ${ }^{8}$. Due to the insufficiency of these prognostic factors, new factors including clinical and laboratory indicators are being considered.

Increasing evidence supports the involvement of systemic nutritional status and inflammation in cancer progression ${ }^{9-11}$. Albumin (ALB) and globulin (GLB) are the two major components of serum proteins. Hypoalbuminemia in cancer patients not only is an indicator of poor nutritional status but also relates to chronic inflammation 12-13. Furthermore, increased level of GLB could serve as a marker of chronic inflammation response and reflect a cumulative exposure of various pro-inflammatory cytokines ${ }^{14}$. Recent studies have demonstrated that ALB to GLB ratio (AGR), which is calculated as AGR= ALB/(total protein-ALB), is an independent prognostic factor for breast cancer, lung cancer, nasopharyngeal carcinoma, colorectal cancer and so on 15-18. AGR level not only reflects the nutritional status but also represents the systemic inflammation. Thus, discriminating AGR might be a potential independent risk factor for RCC. However, to our best knowledge, the prognostic significance of AGR in RCC has not been reported. The aim of our research was to assess the prognostic significance of preoperative AGR in long-term survival of RCC patients and to evaluate whether it could provide additional prognostic information to well-established clinicopathological parameters.

\section{Material and Methods}

\section{Patients}

The subjects of the retrospective study were a cohort of 912 consecutive RCC patients who underwent a curative radical or partial nephrectomy at the Department of Urology in Sun Yat-Sen University Cancer Center between January 2000 and December 2012. Among these patients, 17 patients $(1.86 \%)$ had incomplete laboratory data. Thus, 895 patients were included in the analysis. The study was approved by the Institutional Review Board of Sun Yat-sen University Cancer Center and performed in accordance with the ethical standards of the World Medical Association Declaration of Helsinki ${ }^{19}$. All included patients provided written informed consent and their information were recorded and registered in our cancer registry system.

\section{Follow-up}

Follow-up schedules were established and applied referring to the NCCN Clinical Practice Guidelines. RCC patients at early stage who need to be closely monitored after partial or radical nephrectomy ( $\mathrm{pT} 1 \mathrm{a}$ and $\mathrm{pT} 1 \mathrm{~b}$ ) were subjected to follow-up evaluations every six months for the first 2 years and once a year thereafter. RCC patients at advanced stage were subjected to follow-up evaluations every 3-6 months for the first three years and once a year thereafter. The follow-up evaluations included all routine clinical, laboratory and radiological examinations. In addition, all patients were also followed up via telephone interviews. The last follow-up was completed in November 01, 2015. Patients who were still alive at the last follow-up were censored.

\section{Clinical and laboratory parameters}

All clinicopathological data including demographic parameters, Fuhrman grade, tumor histology, tumor stage and laboratory data were retrieved from the electronic medical records at our hospital. The AJCC/UICC TNM staging system (the $7^{\text {th }}$ edition) was applied to classify the tumor stage. The laboratory data, including the levels of ALB, total proteins, hemoglobin (HGB), alkaline phosphatase (ALP), serum creatinine (Scr) and uric acid (UA), were measured one day before surgical intervention. Serum ALP $>135 \mathrm{U} / \mathrm{L}, \mathrm{Scr}>130 \mu \mathrm{mol} / \mathrm{L}$ and UA $>420$ $\mu \mathrm{mol} / \mathrm{L}$ were defined as elevated. Preoperative AGR was calculated using the equation: AGR = ALB / (total protein- ALB).

\section{Statistical analysis}

The end point of the study was overall survival (OS), which was defined as the time interval between the date of surgery and the date of death of individual patient. The optimal cut-off value of preoperative AGR was determined by using the receiver operating curve (ROC) analysis, as previously described ${ }^{20-21}$. The threshold that best discriminated the differences between the survived and deceased cases (in terms of sensitivity and specificity) was used, allowing us to treat AGR as a dichotomous variable.

The continuous and categorical variables were presented as means \pm standard deviations (SD) as well as frequencies and percentages, respectively. Continuous data were analyzed using the Mann-Whitney U-test and categorical data were analyzed using the chi-square test. Patients' clinical end points were calculated using the Kaplan-Meier method and compared by the log-rank test. Univariate analysis was performed to determine the significance of variables and Cox regression model was performed for OS. Subsequently, the variables with $\mathrm{P}<0.05$ were further analyzed using multivariate Cox proportion analysis to determine independent prognostic factors. Hazard ratios (HRs) estimated 
from the Cox analysis were reported as relative risks with corresponding 95\% confidence intervals (CIs). All Statistical analyses were performed using Empower (R) (www.empowerstats.com, X\&Y solutions, Inc., Boston, MA), R (http://www.R-project.org) and Statistical Package for Social Sciences (SPSS) 21.0 software (IBM, Armonk, NY). A two-sided $\mathrm{P}<0.05$ was considered statistically significant.

Table 1. Baseline characteristics of all patients.

\begin{tabular}{|c|c|c|}
\hline Characteristics & Cases $(\mathrm{n}=895)$ & Percentage (\%) \\
\hline Age, year (mean $\pm S D)$ & $51.44 \pm 13.44$ & \\
\hline Hemoglobin, g/L, (mean \pm SD) & $134.21 \pm 20.47$ & \\
\hline Total protein, g/L (mean $\pm \mathrm{SD})$ & $72.22 \pm 6.61$ & \\
\hline Serum albumin, $\mathrm{g} / \mathrm{L}(\mathrm{mean} \pm \mathrm{SD})$ & $43.10 \pm 4.80$ & \\
\hline Body mass index, $\mathrm{kg} / \mathrm{m}^{2}($ mean $\pm \mathrm{SD})$ & $23.41 \pm 3.58$ & \\
\hline $\mathrm{AGR},($ mean $\pm \mathrm{SD})$ & $1.54 \pm 0.34$ & \\
\hline \multicolumn{3}{|l|}{ Gender } \\
\hline Male & 600 & 67.00 \\
\hline Female & 295 & 33.00 \\
\hline \multicolumn{3}{|l|}{ Pathological types } \\
\hline Clear cell carcinoma & 696 & 77.80 \\
\hline Multilocular cystic renal cell carcinoma & 64 & 7.20 \\
\hline Chromophobe renal carcinoma & 32 & 3.60 \\
\hline Others & 103 & 11.50 \\
\hline \multicolumn{3}{|l|}{ Fuhrman-grade } \\
\hline I & 205 & 22.90 \\
\hline II & 329 & 36.80 \\
\hline III & 85 & 9.50 \\
\hline IV & 13 & 1.50 \\
\hline Unknown & 263 & 29.40 \\
\hline \multicolumn{3}{|l|}{ pT status } \\
\hline T1 & 613 & 68.50 \\
\hline $\mathrm{T} 2$ & 164 & 18.30 \\
\hline T3 & 88 & 9.80 \\
\hline $\mathrm{T} 4$ & 30 & 3.40 \\
\hline \multicolumn{3}{|l|}{$\mathrm{pN}$ status } \\
\hline No & 829 & 92.60 \\
\hline N1 & 66 & 7.40 \\
\hline \multicolumn{3}{|l|}{ pM status } \\
\hline M0 & 852 & 95.20 \\
\hline M1 & 43 & 4.80 \\
\hline \multicolumn{3}{|l|}{ pTNM stage } \\
\hline I & 597 & 66.70 \\
\hline II & 143 & 16.00 \\
\hline III & 96 & 10.70 \\
\hline IV & 59 & 6.60 \\
\hline \multicolumn{3}{|l|}{ Alkaline phosphatase, $\mathrm{U} / \mathrm{L}$} \\
\hline Normal & 858 & 95.90 \\
\hline Elevated & 37 & 4.10 \\
\hline \multicolumn{3}{|l|}{ Serum creatinine, $\mu \mathrm{mol} / \mathrm{L}$} \\
\hline Normal & 859 & 96.00 \\
\hline Elevated & 36 & 4.00 \\
\hline \multicolumn{3}{|l|}{ Uric acid, $\mu \mathrm{mol} / \mathrm{L}$} \\
\hline Normal & 705 & 78.80 \\
\hline Elevated & 190 & 21.20 \\
\hline \multicolumn{3}{|l|}{ AGR group } \\
\hline Low AGR group (AGR $\leq 1.47)$ & 371 & 41.50 \\
\hline High AGR group (AGR > 1.47) & 524 & 58.50 \\
\hline
\end{tabular}

Abbreviation: pTNM: pathologic tumor-node-metastasis; AGR: Albumin to Globulin ratio.

\section{Results}

\section{Clinicopathological Features}

The 895 enrolled patients were $51.44 \pm 13.44$ years old. Of them, $600(67.00 \%)$ were male and 295 $(33.00 \%)$ were female. 597, 143, 96 and 59 patients were at stage I, II, III and IV, respectively. The median follow-up time from diagnosis was 69.68 months (95\% CI: 65.73-73.63). 171 patients died during the follow-up. Table 1 summarizes patients' characteristics.

\section{Identification of the optimal cut-off value for AGR}

Serum ALB and serum total proteins levels were $43.10 \pm 4.80 \mathrm{~g} / \mathrm{L}$ and $72.22 \pm 6.61 \mathrm{~g} / \mathrm{L}$, respectively. The calculated AGR was $1.54 \pm 0.34$. ROC curves in Figure 1 showed that when analyzed as a dichotomous variable, $\mathrm{AGR}=1.47$ as the cut-off value provided the strongest prognostic point for the OS. Therefore, this level was chosen to stratify patients. 371 patients with AGR $\leq 1.47$ were classified into low AGR group, whereas 524 with AGR> 1.47 were classified into high AGR group.

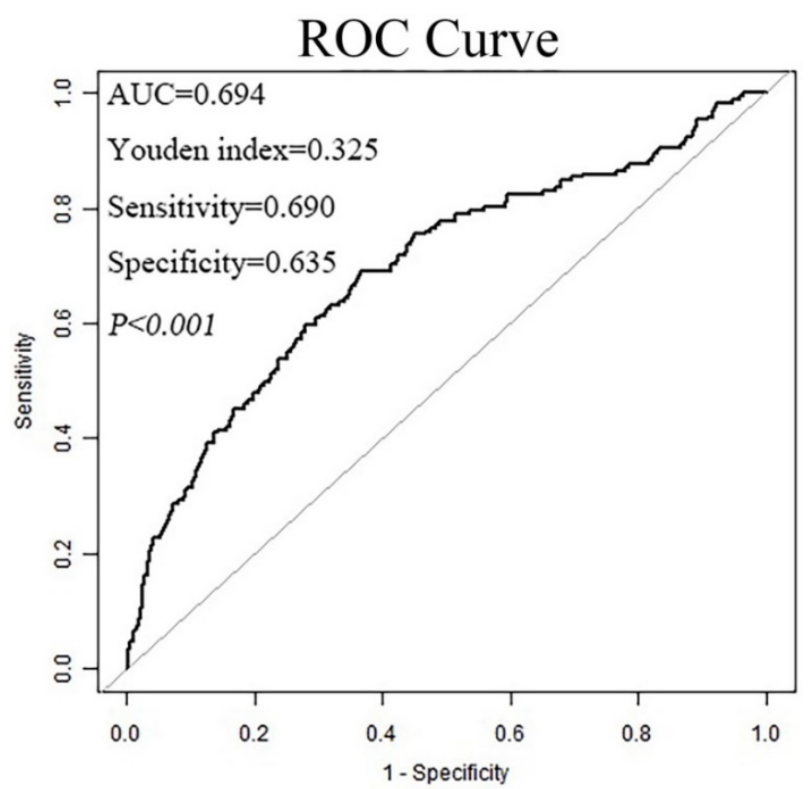

Figure 1. The receiver operating characteristic (ROC) curve for the preoperative albumin to globulin ratio (AGR).

\section{Association of AGR with the clinicopathological features of RCC patients}

Patients in different AGR groups showed significant differences in $\mathrm{pT}$-stage $(\mathrm{P}<0.001)$, $\mathrm{pN}$-stage $(\mathrm{P}<0.001)$, pM-stage $(\mathrm{P}<0.001)$, pTNM-stage $(\mathrm{P}<0.001)$ and ALP group $(\mathrm{P}<0.001)$ (Table 2). Additionally, patients in low AGR group were significantly older $(\mathrm{P}<0.001)$, and had lower HGB $(\mathrm{P}<0.001)$, higher TP 
$(\mathrm{P}<0.001)$, lower ALB $(\mathrm{P}<0.001)$ and lower BMI $(\mathrm{P}<0.001)$ than patients in high AGR group (Table 2).

Table 2. Characteristics of the 895 patients with RCC.

\begin{tabular}{|c|c|c|c|}
\hline Characteristics & Low AGR group & $\begin{array}{l}\text { High AGR } \\
\text { group }\end{array}$ & $P$ value \\
\hline Age, year (mean $\pm S D)$ & $55.20 \pm 13.33$ & $48.78 \pm 12.88$ & $<0.001$ a \\
\hline $\begin{array}{l}\text { Hemoglobin, g/L, } \\
(\text { mean } \pm S D)\end{array}$ & $126.30 \pm 21.40$ & $139.81 \pm 17.79$ & $<0.001^{a}$ \\
\hline Total protein, g/L (mean \pm SD) & $73.97 \pm 6.67$ & $70.98 \pm 6.29$ & $<0.0011^{a}$ \\
\hline $\begin{array}{l}\text { Serum albumin, g/L } \\
(\text { mean } \pm \mathrm{SD})\end{array}$ & $40.46 \pm 5.14$ & $44.97 \pm 3.49$ & $<0.0011^{a}$ \\
\hline $\begin{array}{l}\text { Body mass index, } \mathrm{kg} / \mathrm{m}^{2} \\
(\text { mean } \pm \mathrm{SD})\end{array}$ & $22.94 \pm 3.48$ & $23.74 \pm 3.61$ & $0.001^{a}$ \\
\hline Gender & & & $<0.001 \mathrm{~b}$ \\
\hline Male & $220(59.30 \%)$ & $380(72.50 \%)$ & \\
\hline Female & $151(40.70 \%)$ & $144(27.50 \%)$ & \\
\hline Pathological types & & & $0.075^{b}$ \\
\hline Clear cell carcinoma & $283(76.30 \%)$ & $413(78.80 \%)$ & \\
\hline $\begin{array}{l}\text { Multilocular cystic renal cell } \\
\text { carcinoma }\end{array}$ & $29(7.80 \%)$ & $35(6.70 \%)$ & \\
\hline $\begin{array}{l}\text { Chromophobe renal } \\
\text { carcinoma }\end{array}$ & $8(2.20 \%)$ & $24(4.60 \%)$ & \\
\hline Others & $51(13.70 \%)$ & $52(9.90 \%)$ & \\
\hline Fuhrman-grade & & & $0.066^{\mathrm{b}}$ \\
\hline I & $80(21.60 \%)$ & $125(23.90 \%)$ & \\
\hline II & $125(33.70 \%)$ & $204(38.90 \%)$ & \\
\hline III & $46(12.40 \%)$ & $39(7.40 \%)$ & \\
\hline IV & $7(1.90 \%)$ & $6(1.10 \%)$ & \\
\hline Unknown & $113(30.50 \%)$ & $150(28.60 \%)$ & \\
\hline pT status & & & $<0.001^{b}$ \\
\hline $\mathrm{T} 1$ & $203(54.70 \%)$ & $410(78.20 \%)$ & \\
\hline $\mathrm{T} 2$ & $85(22.90 \%)$ & $79(15.10 \%)$ & \\
\hline $\mathrm{T} 3$ & $60(16.20 \%)$ & $28(5.30 \%)$ & \\
\hline $\mathrm{T} 4$ & $23(6.20 \%)$ & $7(1.30 \%)$ & \\
\hline pN status & & & $<0.001^{b}$ \\
\hline No & $324(87.30 \%)$ & $505(96.40 \%)$ & \\
\hline N1 & $47(12.70 \%)$ & $19(3.60 \%)$ & \\
\hline pM status & & & $<0.001^{b}$ \\
\hline M0 & $339(91.40 \%)$ & $513(97.90 \%)$ & \\
\hline M1 & $32(8.60 \%)$ & $11(2.10 \%)$ & \\
\hline pTNM stage & & & $<0.001^{b}$ \\
\hline I & $196(52.80 \%)$ & $401(76.50 \%)$ & \\
\hline II & $67(18.10 \%)$ & $76(14.50 \%)$ & \\
\hline III & $65(17.50 \%)$ & $31(5.90 \%)$ & \\
\hline IV & $43(11.60 \%)$ & $16(3.10 \%)$ & \\
\hline Alkaline phosphatase, $\mathrm{U} / \mathrm{L}$ & & & $<0.001$ b \\
\hline Normal & $343(92.50 \%)$ & $515(98.30 \%)$ & \\
\hline Elevated & $28(7.50 \%)$ & $9(1.70 \%)$ & \\
\hline Serum creatinine, $\mu \mathrm{mol} / \mathrm{L}$ & & & $0.159 \mathrm{~b}$ \\
\hline Normal & $352(94.90 \%)$ & $507(96.80 \%)$ & \\
\hline Elevated & $19(5.10 \%)$ & $17(3.20 \%)$ & \\
\hline Uric acid, $\mu \mathrm{mol} / \mathrm{L}$ & & & $0.339 \mathrm{~b}$ \\
\hline Normal & $298(80.30 \%)$ & $407(77.70 \%)$ & \\
\hline Elevated & $73(19.70 \%)$ & $117(22.30 \%)$ & \\
\hline
\end{tabular}

Abbreviation: pTNM: pathologic tumor-node-metastasis; AGR: Albumin to Globulin ratio

a Mann-Whitney U-test.

bChi-square test.

\section{Association of preoperative AGR with OS}

Kaplan-Meier survival analysis showed that patients in low AGR group had significantly poorer OS than patients in high AGR group (mean OS: low AGR vs high AGR, 114.01 vs 151.51 months, log-rank
$\mathrm{P}<0.001$; Figure 2). The univariate analysis revealed that preoperative high AGR was associated with decreased risk of death (HR: 0.29, 95\% CI: 0.21-0.40, $\mathrm{P}<0.001$, Table 3). Therefore, a multivariate analysis using Cox proportional hazards model for OS was performed to determine various prognostic indicators including age, HGB, BMI, pathology, Fuhrman grade, pT-stage, pN-stage, pM-stage, ALP and AGR. The results showed that preoperative AGR was an independent prognostic indicator of OS (HR: 0.63, 95\%CI: 0.43-0.93, $\mathrm{P}=0.022$, Table 3 ). In addition, serum ALB, $\mathrm{pT}$-stage, $\mathrm{pN}$-stage and $\mathrm{pM}$-stage were also identified as independent prognostic indicators for OS.

\section{Association of preoperative AGR with subgroups}

The prognostic influence of preoperative AGR in subgroups of $\mathrm{pT}$-stage, $\mathrm{pN}$-stage and $\mathrm{pM}$-stage were further investigated. Patients with low AGR level had a significantly shorter OS than patients with high AGR level in pT1-2 subgroup (mean OS: low AGR vs high AGR, 127.67 vs 162.92 months, log-rank $\mathrm{P}<0.001$; Figure 3A), pT3-4 subgroup (mean OS: low AGR vs high AGR, 67.29 vs 101.12 months, log-rank $\mathrm{P}=0.010$; Figure 3B), $\mathrm{pN} 0$ subgroup (mean OS: low AGR vs high AGR, 125.45 vs 153.87 months, log-rank $\mathrm{P}<0.001$; Figure $3 \mathrm{C}$ ), $\mathrm{pN} 1$ subgroup (mean OS: low AGR vs high AGR, 38.80 vs 74.23 months, log-rank $\mathrm{P}=0.007$; Figure 3D), pM0 subgroup (mean OS: low AGR vs high AGR, 123.11 vs 152.67 months, log-rank $P<0.001$; Figure 3E) and pM1 subgroup (mean OS: low AGR vs high AGR, 29.25 vs 52.02 months, log-rank $\mathrm{P}=0.033$; Figure 3F).

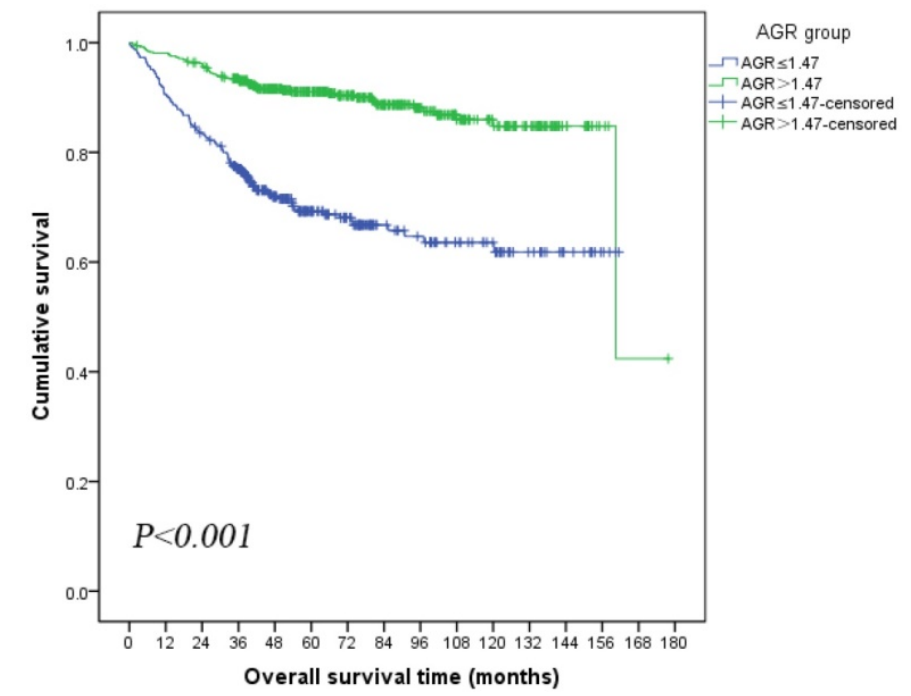

Figure 2. Kaplan-Meier curves depicting overall survival (OS) according to the preoperative optimal value of albumin to globulin ratio (AGR) in patients with renal cell carcinoma. 

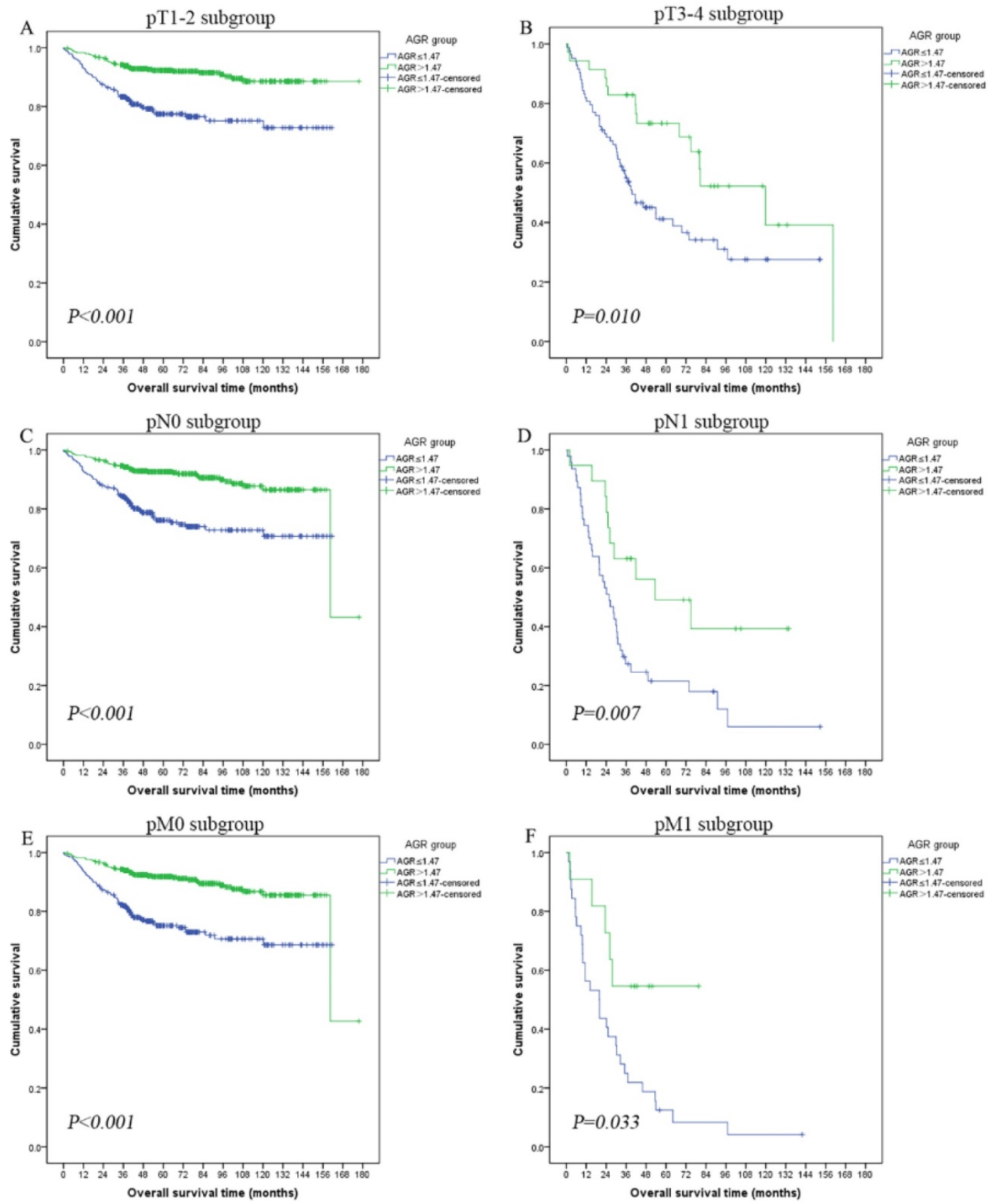

Figure 3. Kaplan-Meier curves showing overall survival (OS) according to the preoperative optimal value of albumin to globulin ratio (AGR) in all patients with renal cell carcinoma. Patients were stratified according to the $\mathrm{pT}$-status, pN-status, and pM-status. (A) Kaplan-Meier analysis of OS in pTI-2 subgroup. (B) Kaplan-Meier analysis of OS in PT3-4 subgroup. (C) Kaplan-Meier analysis of OS in pNO subgroup. (D) Kaplan-Meier analysis of OS in pNI subgroup. (E) Kaplan-Meier analysis of OS in PMO subgroup. (F) Kaplan-Meier analysis of OS in PMI subgroup.

Table 3. Correlation of basic characteristics in all patients to the overall survival (OS) by Cox regression analyses.

\begin{tabular}{|c|c|c|c|c|c|c|}
\hline \multirow[b]{2}{*}{ Predictors } & \multicolumn{3}{|c|}{ univariate analyses } & \multicolumn{3}{|c|}{ multivariate analyses } \\
\hline & HR & $95 \% \mathrm{CI}$ & Pvalue & HR & $95 \% \mathrm{CI}$ & P value \\
\hline Age & 1.02 & 1.01 to 1.03 & 0.003 a & 1.00 & 0.99 to 1.02 & $0.138^{b}$ \\
\hline Hemoglobin & 0.98 & 0.98 to 0.99 & $<0.001^{\text {a }}$ & 0.99 & 0.98 to 1.00 & $0.063^{b}$ \\
\hline Total protein & 1.00 & 0.98 to 1.02 & $0.854^{a}$ & & & \\
\hline Serum albumin & 0.92 & 0.90 to 0.94 & $<0.001$ a & 0.95 & 0.92 to 0.99 & $0.015^{b}$ \\
\hline Body mass index & 0.89 & 0.85 to 0.93 & $<0.001$ a & 0.95 & 0.90 to 1.00 & $0.054^{\mathrm{b}}$ \\
\hline \multicolumn{7}{|l|}{ Gender } \\
\hline Male & 1.00 & & & & & \\
\hline
\end{tabular}




\begin{tabular}{|c|c|c|c|c|c|c|}
\hline \multirow[b]{2}{*}{ Predictors } & \multicolumn{3}{|c|}{ univariate analyses } & \multicolumn{3}{|c|}{ multivariate analyses } \\
\hline & $\overline{\mathrm{HR}}$ & $95 \% \mathrm{CI}$ & P value & HR & $95 \% \mathrm{CI}$ & P value \\
\hline Female & 0.90 & 0.65 to 1.25 & $0.529 \mathrm{a}$ & & & \\
\hline \multicolumn{7}{|l|}{ Pathological types } \\
\hline Clear cell carcinoma & 1.00 (ref.) & & & 1.00 (ref.) & & \\
\hline Multilocular cystic renal cell carcinoma & 2.48 & 1.58 to 3.87 & $<0.001^{\text {a }}$ & 1.58 & 0.93 to 2.69 & $0.089 \mathrm{~b}$ \\
\hline Chromophobe renal carcinoma & 0.19 & 0.03 to 1.40 & 0.103 a & 0.22 & 0.03 to 1.67 & $0.146^{b}$ \\
\hline Others & 1.63 & 1.08 to 2.46 & $0.020^{a}$ & 0.97 & 0.61 to 1.54 & $0.918^{b}$ \\
\hline \multicolumn{7}{|l|}{ Fuhrman-grade } \\
\hline I & 1.00 (ref.) & & & 1.00 (ref.) & & \\
\hline II & 1.16 & 0.73 to 1.86 & $0.525^{\mathrm{a}}$ & 1.16 & 0.72 to 1.88 & $0.529 \mathrm{~b}$ \\
\hline III & 2.76 & 1.61 to 4.73 & $<0.001^{\mathrm{a}}$ & 1.66 & 0.94 to 2.93 & $0.079^{b}$ \\
\hline IV & 4.75 & 1.96 to 11.48 & $<0.001^{\mathrm{a}}$ & 2.23 & 0.87 to 5.70 & $0.092^{b}$ \\
\hline Unknown & 1.94 & 1.25 to 3.00 & $0.003^{a}$ & 1.60 & 1.00 to 2.56 & $0.047^{b}$ \\
\hline \multicolumn{7}{|l|}{ pT status } \\
\hline $\mathrm{T} 1$ & 1.00 (ref.) & & & 1.00 (ref.) & & \\
\hline $\mathrm{T} 2$ & 2.33 & 1.57 to 3.46 & $<0.001{ }^{a}$ & 1.52 & 0.99 to 2.33 & $0.053^{b}$ \\
\hline $\mathrm{T} 3$ & 5.39 & 3.66 to 7.93 & $<0.001$ a & 2.34 & 1.51 to 3.62 & $<0.001^{b}$ \\
\hline $\mathrm{T} 4$ & 11.60 & 7.20 to 18.67 & $<0.001^{\mathrm{a}}$ & 1.75 & 0.91 to 3.36 & $0.091^{b}$ \\
\hline \multicolumn{7}{|l|}{$\mathrm{pN}$ status } \\
\hline N0 & 1.00 (ref.) & & & 1.00 (ref.) & & \\
\hline N1 & 8.21 & 5.87 to 11.50 & $<0.001^{\text {a }}$ & 3.05 & 1.97 to 4.74 & $<0.001^{b}$ \\
\hline \multicolumn{7}{|l|}{ pM status } \\
\hline M0 & 1.00 (ref.) & & & 1.00 (ref.) & & \\
\hline M1 & 9.84 & 6.74 to 14.36 & $<0.001^{\mathrm{a}}$ & 3.49 & 2.19 to 5.57 & $<0.001^{b}$ \\
\hline \multicolumn{7}{|l|}{ pTNM stage } \\
\hline I & 1.00 (ref.) & & & & & \\
\hline II & 1.91 & 1.19 to 3.07 & $0.007^{a}$ & & & \\
\hline III & 6.25 & 4.21 to 9.26 & $<0.001^{\text {a }}$ & & & \\
\hline IV & 15.62 & 10.46 to 23.31 & $<0.0011^{a}$ & & & \\
\hline \multicolumn{7}{|l|}{ Alkaline phosphatase } \\
\hline Normal & 1.00 (ref.) & & & 1.00 (ref.) & & \\
\hline Elevated & 2.33 & 1.36 to 3.97 & $0.001^{\mathrm{a}}$ & 0.54 & 0.29 to 1.00 & $0.050^{b}$ \\
\hline \multicolumn{7}{|l|}{ Serum creatinine } \\
\hline Normal & 1.00 (ref.) & & & & & \\
\hline Elevated & 1.66 & 0.92 to 2.99 & $0.092^{\mathrm{a}}$ & & & \\
\hline \multicolumn{7}{|l|}{ Uric acid } \\
\hline Normal & 1.00 (ref.) & & & & & \\
\hline Elevated & 1.15 & 0.80 to 1.65 & $0.455^{\mathrm{a}}$ & & & \\
\hline \multicolumn{7}{|l|}{ AGR group } \\
\hline Low AGR group & 1.00 (ref.) & & & 1.00 (ref.) & & \\
\hline High AGR group & 0.29 & 0.21 to 0.40 & $<0.001^{a}$ & 0.63 & 0.43 to 0.93 & $0.022^{b}$ \\
\hline
\end{tabular}

\section{Discussion}

In our study, we demonstrated that preoperative AGR could be used as a prognostic factor of OS for RCC patients. Although several studies have shown a relationship between AGR and prognosis of patients with various types of cancers 15-18, 21, to our best knowledge, this is the first study to assess the value of preoperative AGR as a prognostic maker for predicting OS of patients with RCC.

Serum ALB and GLB are two main constituents of serum total protein and can be easily and cost-effectively measured, thus providing a simple means of estimating visceral protein function. Serum ALB is a hepatic protein with a half-life of 14-20 days. It functions as a carrier molecule for various minerals, hormones and fatty acids and also helps to maintain oncotic pressure in capillaries ${ }^{22}$. In addition, Serum
ALB is characterized as a negative acute-phase protein, and its pool is affected by a number of inflammatory conditions 23. Besides, a previous research revealed that serum ALB inhibited the proliferation of human breast cancer cell lines by modulating the activities of autocrine growth regulatory factors in vitro ${ }^{24}$. Moreover, albumin synthesis might be suppressed by malnutrition and systematic inflammation, resulting in hypoalbuminemia, which could weaken human immune defense mechanisms. Recent studies have revealed that hypoalbuminemia was more attributed to systemic inflammation than malnutrition in the dialysis patients ${ }^{25}$. As part of cancer-related systemic inflammation, activation of proinflammatory cytokines such as IL-6 and tumor necrotic factor-a could lead to low serum ALB concentration ${ }^{26}$. In addition, elevated accumulation of acute phase 
proteins, immunoglobulins as well as other serum proteins may increase serum GLB level. These changes were reflective of an inflammatory state. Some researchers have demonstrated that serum GLB is associated with the prognosis of cancer patients $27-28$.

Taken together, we hypothesized the AGR is a more accurate prognostic indicator than serum ALB or GLB alone. A large retrospective cohort study demonstrated that low AGR level was associated with an increased risk for cancer incidence and mortality in both short- and long-term follow-up in generally healthy population ${ }^{29}$. Although cancer types are implicated, low AGR level is widely accepted to be linked to tumor progression ${ }^{30}$. Based on observations that cancer often rises at the site of chronic inflammation and inflammatory cells are present in tumor sites ${ }^{31}$, this phenomenon may be caused by chronic inflammation. We believe that nutritional status and systemic inflammatory response play major roles in the progression of RCC and preoperative AGR could be a prognostic indicator for RCC patients.

In this large retrospective cohort study, a cut-off preoperative AGR value of 1.47 was used for predicting the OS of RCC patients. The results showed that preoperative AGR $\leq 1.47$ was associated with older age population, low HGB, low BMI, low ALB and advanced RCC stage. The reason is that low preoperative AGR was associated with malnutrition and inflammatory response, which was consistent with that weight loss and ongoing inflammation-like response contribute to the subsequent death of RCC patients, especially in advanced stages. According to the Kaplan-Meier curves and log-rank test, patients with low preoperative AGR level had significantly shorter OS than patients with high AGR level. Furthermore, the univariate and multivariate Cox regression models revealed that AGR was a significant independent predictor for OS of RCC patients subjected to surgery. Besides, subgroup analysis of patients at pT1-2, pT3-4, pN0, pN1, pM0 and pM1 stages showed that patients with low preoperative AGR had significantly shorter OS than patients with high preoperative AGR. For these reasons, we believe that preoperative AGR is an independent prognostic factor for RCC patients.

The study has several limitations. Firstly, some specific inflammatory markers such as C-reactive protein and cytokine levels, which were also important factors of inflammation, were not measured because they were not routinely measured at our hospital prior to 2006. Secondly, preoperative AGR was only assessed at a single time point before surgery and in a single center. Further large-scale population-based prospective studies are needed to fully consolidate the results.

Despite the aforementioned limitations, our study had some clinical implications. First of all, we are the first to show that low preoperative AGR may be a clinical indicator associated with shorter OS of RCC patients. Secondly, AGR is routinely measured at low cost in clinical practice, thus it has potential as a simple, convenient predictive and stratification factor to assist with clinical decision-making for RCC patients. Thirdly, at least in theory, AGR is a predictive factor superior to other nutritional or inflammatory indicators. Nutritional assessment and support as well as anti-inflammatory therapy may be suitable treatment choices for patients with AGR $\leq 1.47$. Although Algra et al. have demonstrated the ability of anti-inflammatory therapy (e.g., aspirin and other non-steroidal anti-inflammatory drugs) to prevent and/or treat some cancers ${ }^{32}$, the value of anti-inflammatory therapy for RCC patients needs to be further studied. At least, AGR can provide a useful tool for future clinical trials and patient management.

In conclusion, preoperative AGR is a proven objective, reproducible and inexpensive predictor of survival of RCC patients subjected to surgical resection and consideration should be given for its routine clinical use.

\section{Implications for Practice}

The serum albumin to globulin ratio (AGR) not only reflects patients' nutritional status but also represents their systemic inflammation. This prospective cohort study of 895 patients with renal cell carcinoma (RCC) showed that preoperative AGR was an independent prognostic indicator of overall survival (OS) of RCC patients.

\section{Acknowledgements}

This study was supported by grants from National Natural Science Foundation of China (Grant No. 81302224 and 81202013), China Postdoctoral Science Foundation (Grant No. 2016M592582), Natural Science Foundation of Guangdong Province (Grant No. 2016A030610219) and Medical Scientific Research Foundation of Guangdong Province, China (Grant No. B2012131). The funders had no role in study design, data collection and analysis, decision to publish, or preparation of the manuscript.

\section{Author Contributions}

Conception and Design: YK, HXB, GSJ.

Provision of Study Material or Patients: $Y K$, HXB, GSJ, CD, HH.

Collection and Assembly of Data: YK, HXB, GSJ, CD, CX, ZYJ, HQM, XYF,

Data Analysis and Interpretation: YK, HXB, 
GSJ, YGW, QZK, LZW, ZM, LRW, ZFJ.

Manuscript Writing: YK, HXB, GSJ, CD, LRW,

ZFJ, HH.

Final Approval of Manuscript: All the authors.

\section{Conflicts of interest} interest.

All the authors state they have no conflicts of

\section{References}

1. Chen W, Zheng R, Baade PD, et al. Cancer statistics in China, 2015. CA Cancer J Clin 2016; 66:115-132.

2. Siegel RL, Miller KD, Jemal A. Cancer statistics, 2016. CA Cancer J Clin 2016; 66:7-30.

3. Jayson M, Sanders H. Increased incidence of serendipitously discovered renal cell carcinoma. UROLOGY 1998; 51:203-205.

4. Luciani LG, Cestari R, Tallarigo C. Incidental renal cell carcinoma-age and stage characterization and clinical implications: study of 1092 patients (1982-1997). UROLOGY 2000; 56:58-62.

5. Eggener SE, Yossepowitch O, Pettus JA, et al. Renal cell carcinoma recurrence after nephrectomy for localized disease: predicting survival from time of recurrence. J CLIN ONCOL 2006; 24:3101-3106.

6. Kim SP, Alt AL, Weight CJ, et al. Independent validation of the 2010 American Joint Committee on Cancer TNM classification for renal cell carcinoma: results from a large, single institution cohort. J Urol 2011; 185:2035-2039.

7. Fuhrman SA, Lasky LC, Limas C. Prognostic significance of morphologic parameters in renal cell carcinoma. AM J SURG PATHOL 1982; 6:655-663.

8. Volpe A, Patard JJ. Prognostic factors in renal cell carcinoma. WORLD J UROL 2010; 28:319-327.

9. Delmore G. Assessment of nutritional status in cancer patients: widely neglected? SUPPORT CARE CANCER 1997; 5:376-380.

10. McMillan DC. Systemic inflammation, nutritional status and survival in patients with cancer. Curr Opin Clin Nutr Metab Care 2009; 12:223-226.

11. Coussens LM, Werb Z. Inflammation and cancer. NATURE 2002; 420:860-867.

12. McMillan DC, Watson WS, O'Gorman P, et al. Albumin concentrations are primarily determined by the body cell mass and the systemic inflammatory response in cancer patients with weight loss. NUTR CANCER 2001; 39:210-213.

13. Gupta D, Lis CG. Pretreatment serum albumin as a predictor of cancer survival: a systematic review of the epidemiological literature. NUTR J 2010; 9:69.

14. Gabay C, Kushner I. Acute-phase proteins and other systemic responses to inflammation. N Engl J Med 1999; 340:448-454.

15. Azab BN, Bhatt VR, Vonfrolio S, et al. Value of the pretreatment albumin to globulin ratio in predicting long-term mortality in breast cancer patients. $A M J$ SURG 2013; 206:764-770.

16. Zhou T, He X, Fang W, et al. Pretreatment Albumin/Globulin Ratio Predicts the Prognosis for Small-Cell Lung Cancer. Medicine (Baltimore) 2016; 95:e3097.

17. Du XJ, Tang LL, Mao YP, et al. The pretreatment albumin to globulin ratio has predictive value for long-term mortality in nasopharyngeal carcinoma. PLOS ONE 2014; 9:e94473.

18. Azab B, Kedia $\mathrm{S}$, Shah $\mathrm{N}$, et al. The value of the pretreatment albumin/globulin ratio in predicting the long-term survival in colorectal cancer. INT J COLORECTAL DIS 2013; 28:1629-1636.

19. World Medical Association Declaration of Helsinki: ethical principles for medical research involving human subjects. J Am Coll Dent 2014; 81:14-18.

20. Absenger G, Szkandera J, Pichler M, et al. A derived neutrophil to lymphocyte ratio predicts clinical outcome in stage II and III colon cancer patients. $\mathrm{Br}$ J Cancer 2013; 109:395-400.

21. Zhang B, Yu W, Zhou LQ, et al. Prognostic Significance of Preoperative Albumin-Globulin Ratio in Patients with Upper Tract Urothelial Carcinoma. PLOS ONE 2015; 10:e144961.

22. Doweiko JP, Nompleggi DJ. The role of albumin in human physiology and pathophysiology, Part III: Albumin and disease states. JPEN J Parenter Enteral Nutr 1991; 15:476-483.

23. Bharadwaj S, Ginoya S, Tandon P, et al. Malnutrition: laboratory markers vs nutritional assessment. Gastroenterol Rep $(O x f) 2016$.

24. Laursen I, Briand P, Lykkesfeldt AE. Serum albumin as a modulator on growth of the human breast cancer cell line, MCF-7. ANTICANCER RES 1990; 10:343-351.

25. Kaysen GA. Serum albumin concentration in dialysis patients: why does it remain resistant to therapy? KIDNEY INT SUPPL 2003;:S92-S98.

26. Seaton K. Albumin concentration controls cancer. J NATL MED ASSOC 2001; 93:490-493.

27. Adly L, Hill D, Sherman ME, et al. Serum concentrations of estrogens, sex hormone-binding globulin, and androgens and risk of breast cancer in postmenopausal women. INT J CANCER 2006; 119:2402-2407.

28. Guthrie GJ, Roxburgh CS, Farhan-Alanie OM, et al. Comparison of the prognostic value of longitudinal measurements of systemic inflammation in patients undergoing curative resection of colorectal cancer. Br J Cancer 2013; 109:24-28.

29. Suh B, Park S, Shin DW, et al. Low albumin-to-globulin ratio associated with cancer incidence and mortality in generally healthy adults. ANN ONCOL 2014; 25:2260-2266.

30. Coussens LM, Werb Z. Inflammation and cancer. NATURE 2002; 420:860-867.

31. Mantovani A, Allavena $\mathrm{P}$, Sica A, et al. Cancer-related inflammation. NATURE 2008; 454:436-444.

32. Algra AM, Rothwell PM. Effects of regular aspirin on long-term cancer incidence and metastasis: a systematic comparison of evidence from observational studies versus randomised trials. LANCET ONCOL 2012; 13:518-527. 